\title{
Evolução temporal e caracterização dos casos de sífilis congênita em Minas Gerais, Brasil, 2007-2015
}

\author{
Temporal evolution and characterization of congenital syphilis \\ cases in Minas Gerais, Brazil, 2007-2015
}

Patrícia Iolanda Coelho Alves (https://orcid.org/0000-0002-4003-6951) ${ }^{1}$

Lúcia Marina Scatena (https://orcid.org/0000-0002-4356-4557) ${ }^{2}$

Vanderlei José Haas (https://orcid.org/0000-0001-8743-0123) ${ }^{1}$

Sybelle de Souza Castro (https://orcid.org/0000-0002-0005-7555) ${ }^{2}$
${ }^{1}$ Programa de PósGraduação em Atenção à Saúde, Universidade Federal do Triângulo Mineiro (UFTM). Av. Getúlio Guaritá 107, Abadia. 38025440 Uberaba MG Brasil. sybelle.castro@uftm.edu.br ${ }^{2}$ Departamento DidáticoCientífico de Saúde

Coletiva, UFTM. Uberaba MG Brasil.
Abstract The objective was to analyze the temporal trend of the incidence of congenital syphilis (CS) and to characterize the disease in the state of Minas Gerais (MG) between 2007 and 2015. Quantitative study, of a time series analysis, with the database from the Information System of Diseases Notification, referring to cases of CS reported in MG between 2007 and 2015. It was used descriptive statistics, calculation of the incidence rate of CS and polynomial regression model for temporal trend analysis. The results showed that in the period 4,381 cases were registered. The incidence rate ranged from 0.61 to 5.08 per 1,000 live births $(L B)$, with an increase in the temporal trend of the CS incidence coefficient in the period from 2007 to 2015, with an annual rate variation of $30.6 \%$ (95\% CI: 21.0 - 41.0). There was a predominance of brown-skinned newborns (38.7\%), up to 6 days old (94.7\%) and the majority (63.6\%) did not present any suggestive manifestations of CS, but definitive diagnosis for recent CS (95.2\%). The number of reported cases is increasing, suggesting that it is necessary, for improvement in the prenatal care, diagnosis, appropriate treatment, health care and notification.

Key words Syphilis, Congenital syphilis, Pregnant women, Sexually transmitted diseases, Time series studies
Resumo Objetivou-se analisar a tendência temporal da incidência de sifilis congênita (SC) e caracterizar a doença no estado de Minas Gerais (MG) entre 2007 e 2015. Estudo quantitativo, de análise de série temporal, com banco de dados do Sistema de Informação de Agravos de Notificação, referentes aos casos de SC notificados em MG entre 2007 e 2015. Utilizou-se estatística descritiva, cálculo da taxa de incidência de SC e modelo de regressão polinomial para análise de tendência temporal. Os resultados mostraram que no periodo registraram-se 4.381 casos. A taxa de incidência variou entre 0,61 e 5,08/1.000 nascidos vivos $(\mathrm{NV})$, com incremento na tendência temporal do coeficiente de incidência da SC no período de 2007 a 2015, com variação anual da taxa de 30,6\% (IC95\%: 21,0 - 41,0). Houve predominio de recém-nascidos de cor de pele parda (38,7\%), faixa etária de até 6 dias de vida (94,7\%) e a maioria $(63,6 \%)$ não apresentou, em relação ao exame físico, qualquer manifestação sugestiva de SC, porém teve diagnóstico definitivo para SC recente (95,2\%). O número de casos notificados é crescente, sugerindo que há uma necessidade de melhoria na assistência ao pré-natal, diagnóstico, tratamento adequado, ações de saúde e notificação.

Palavras-chave Sifilis, Sifilis congênita, Gestantes, Doenças sexualmente transmissiveis, Estudos de séries temporais 


\section{Introdução}

A sífilis em gestante (SG) é uma doença evitável e de fácil diagnóstico, porém, ainda é considerada um importante problema de saúde pública devido à alta transmissibilidade e frequência, o que leva a complicações graves ${ }^{1,2}$. A sífilis congênita (SC) é uma doença transmitida ao feto pela mãe infectada não tratada ou inadequadamente tratada, por meio da circulação transplacentária ${ }^{3-5}$. A infecção fetal geralmente ocorre entre a $16^{\mathrm{a}}$ e a $28^{\mathrm{a}}$ semana de gestação ${ }^{6}$. O não tratamento da infecção materna recente implica contaminação do feto em 80 a $100 \%$ dos $\operatorname{casos}^{7}$. O diagnóstico oportuno da sífilis na gravidez é o principal desafio para o controle da SC e suas complicações durante e pós-gestação, por exemplo, parto prematuro, óbito fetal e neonatal ${ }^{8,9}$. A ocorrência de SC é reconhecida como um indicador sensível de avaliação da qualidade da assistência pré-natal $(\mathrm{PN})^{10}$.

Considerando a SC grave e potencialmente fatal, diretrizes brasileiras preconizam que toda gestante seja submetida a, no mínimo, dois exames para diagnóstico da sífilis durante o $\mathrm{PN}$, o Venereal Disease Research Laboratory (VDRL). Normalmente o primeiro no início da gestação (idealmente no primeiro trimestre) e o outro aproximadamente na vigésima oitava semana de gestação (início do terceiro trimestre) ${ }^{11}$. Deve-se ainda realizar um VDRL no momento do parto, a fim de proporcionar ao recém-nascido $(\mathrm{RN})$ a possibilidade de tratamento precoce, no caso da gestante não ter sido tratada ou ter sido reinfectada após tratamento ${ }^{4,7}$.

No Brasil, entre janeiro de 1998 e junho de 2017, foram notificados 159.890 casos de SC em menores de um ano de idade, dos quais 70.558 $(44,1 \%)$ eram residentes na Região Sudeste. Quanto à mortalidade infantil por SC, no período de 1998 a 2016, o número de óbitos declarados no Sistema de Informação sobre Mortalidade (SIM) foi de 2.102, sendo 910 (43,3\%) na Região Sudeste ${ }^{12}$.

A importância de analisar a tendência temporal da SC em Minas Gerais (MG) decorre da escassez de estudos sobre este aspecto no estado. Apesar de ser uma patologia de fácil tratamento e prevenção, estudos em distintas regiões brasileiras mostram incremento significativo da incidência ${ }^{13,14}$. É relevante o conhecimento do comportamento da tendência no decorrer dos anos, visando identificar características clínicas e epidemiológicas, reconhecer os nós críticos das intervenções à saúde materno-infantil, com o intuito de otimizar o diagnóstico e o tratamento precoces para a redução de complicações decor- rentes desta doença, assim como atingir a meta de redução da incidência de SC proposta pela Organização Mundial da Saúde (OMS) que é de menos 0,5 caso/1000 NV.

Pesquisas com utilização de base de dados estaduais são necessárias para avaliação da efetividade das ações de saúde e dos Sistemas de Informação em Saúde (SIS), especialmente no diagnóstico e planejamento, tendo em vista a inserção contínua e semanal dos dados nos sistemas pelos serviços de atenção à saúde. Este estudo, portanto, pretende analisar a tendência temporal dos casos de SC e caracterizar a doença no estado de MG entre 2007 e 2015.

\section{Métodos}

Trata-se de um estudo quantitativo, retrospectivo e de análise de série temporal. A pesquisa foi desenvolvida com os casos de SC de indivíduos notificados e residentes no estado de MG entre 2007 e 2015. Utilizou-se o banco de dados do Sistema de Informação de Agravos de Notificação (SINAN) fornecido pela Secretaria de Estado de Saúde de Minas Gerais (SES/MG). O Estado de MG é o segundo mais populoso do Brasil, com 21,1 milhões de habitantes.

Foram excluídos variáveis com incompletude (dados ignorados ou em branco) acima de $20 \%$, porém algumas variáveis relevantes foram analisadas com parcimônia quando acima deste porcentual estabelecido. Para a análise da completude do preenchimento das variáveis do banco de dados utilizou-se os critérios propostos pela Comissão Econômica para a América Latina e o Caribe (CEPAL), que considera a proporção de informação ignorada, os campos em branco e os códigos atribuídos como informação ignorada especificada, caracterizados como incompletude de informação. A CEPAL considera excelente quando há menos de $5 \%$ de informações incompletas, bom de $5 \%$ a $10 \%$, regular de $10 \%$ a $20 \%$, ruim de $20 \%$ a $50 \%$ e muito ruim de $50 \%$ ou mais ${ }^{15}$. Utilizou-se o primeiro e o último ano da série para fins de comparação da completude, 2007 e 2015 respectivamente.

Realizaram-se análises exploratórias descritivas dos dados, a partir de frequência simples absolutas e percentuais para as variáveis categóricas. Os dados foram analisados no programa estatístico Statiscal Package for Social Sciences (SPSS ${ }^{\circledR}$ ) versão 21.0. Calculou-se o coeficiente de incidência por estratos etários e por ano (2007 a 2015), expresso como número de casos por 1.000 $\mathrm{NV} /$ ano, conforme a fórmula ${ }^{12}$ : 
Taxa de incidência de sífilis congênita $=\frac{n^{\circ} \text { casos novos de sífilis congênita no ano }}{n^{\circ} \text { total de nascidos vivos no ano }} \times 1.000$

Para a análise da tendência temporal foi utilizado o modelo de regressão linear generalizada de Prais-Winsten ${ }^{16}$. Foi determinada a taxa de variação e o respectivo intervalo de confiança (IC95\%). Considera-se taxa estável quando o coeficiente de regressão não apresentar diferença significativa ( $p>0,05)$, ascendente quando a mudança anual for positiva e descendente quando negativa.

\section{Resultados}

Em MG, entre 2007 e 2015, houveram 4.381 casos notificados de SC. Observou-se que a incidência da infecção aumentou no período. A taxa de incidência variou entre 0,61 e 5,08 por 1.000 NV no período (Tabela 1 ).

Foi verificada a qualidade do preenchimento do formulário de notificação, com intuito de selecionar as variáveis adequadas para análise, selecionou-se o ano de 2015, por ser o mais recente. Tiveram classificação 'excelente' $(<5 \%$ de incompletude) as variáveis: se a gestante realizou teste não treponêmico (TNT) no parto/curetagem e TNT do sangue periférico da criança. Foram classificadas como completude 'boa' ou 'regular' as variáveis: zona da residência, realização de PN na gestação, diagnóstico de sífilis materna, diagnóstico clínico, presença de sinais/ sintomas, esquema de tratamento da criança, evolução do caso (criança) e titulação, realização de teste treponêmico (TT) confirmatório no parto/curetagem e esquema de tratamento da mãe e TNT do líquor da criança. Dentre as variáveis classificadas como 'ruim', destacam-se raça/cor, escolaridade, se o parceiro foi tratado concomitantemente à gestante, realização de TT (após 18 meses) na criança, evidência de T. pallidum, alteração liquórica e diagnóstico radiológico da criança. Classificada como 'muito ruim', ocupação da mãe (dados não apresentados em tabela). Com exceção de poucas variáveis o banco pode ser considerado adequado para a análise.

Considerando a caracterização sociodemográfica observou-se que a maioria era residente em zona urbana, 4.025 casos (91,9\%); faixa etária de maior prevalência de 0 a 6 dias $(94,7 \%)$, praticamente não houve diferença entre sexos, 1.990 casos (47,9\%) femininos e 1.993 (48,0\%) masculinos (Tabela 2).

No período, 2.788 crianças $(63,6 \%)$ não apresentaram qualquer manifestação clínica sugestiva de SC em relação ao exame físico, apresentaram apenas 614 (14,0\%). Evoluíram como vivos no momento da notificação $3.730(85,1 \%)$ crianças, 115 (2,6\%) foram a óbito por SC, 44 $(1,0 \%)$ a óbito por outras causas e $165(3,8 \%)$ foram abortos. Segundo o estadiamento do caso, foram diagnosticados como SC recente 95,2\% dos casos; $0,1 \%$ como SC tardia e $4,7 \%$ como natimorto com sífilis (Tabela 3 ).

Tabela 1. Distribuição da frequência, proporção e incidência dos casos (por 1.000 NV) de Sífilis Congênita, no estado de Minas Gerais, 2007 a 2015. $(\mathrm{n}=4.381)$.

\begin{tabular}{lcccc}
\hline Ano diagnóstico & No de NV & $\begin{array}{c}\text { No de casos } \\
\text { notificados }\end{array}$ & \% & Incidência \\
\hline 2007 & 260.666 & 159 & 3,6 & 0,61 \\
2008 & 261.626 & 189 & 4,3 & 0,72 \\
2009 & 253.700 & 189 & 4,3 & 0,74 \\
2010 & 255.443 & 224 & 5,1 & 0,88 \\
2011 & 260.037 & 289 & 6,6 & 1,11 \\
2012 & 260.744 & 479 & 10,9 & 1,84 \\
2013 & 258.885 & 616 & 14,1 & 2,38 \\
2014 & 267.075 & 873 & 19,9 & 3,27 \\
2015 & 268.126 & 1363 & 31,1 & 5,08 \\
Total & 2.346 .302 & 4381 & 100,0 & 1,9 \\
\hline
\end{tabular}

Fonte: Elaborado pela autora, 2016. Dados do SINAN E SINASC: 2007 - 2015, obtidos na Secretaria de Estado de Saúde/MG. 
Tabela 2. Caracterização sociodemográfica dos casos de Sífilis Congênita, no estado de Minas Gerais, 2007 a 2015. $(n=4.381)$.

\begin{tabular}{|c|c|c|c|c|c|c|c|c|c|c|}
\hline & \multicolumn{2}{|c|}{2007} & \multicolumn{2}{|c|}{2009} & \multicolumn{2}{|c|}{2011} & \multicolumn{2}{|c|}{2013} & \multicolumn{2}{|c|}{2015} \\
\hline & $\mathbf{N}$ & $\%$ & $\mathbf{N}$ & $\%$ & $\mathbf{N}$ & $\%$ & $\mathbf{N}$ & $\%$ & $\mathbf{N}$ & $\%$ \\
\hline \multicolumn{11}{|l|}{ Faixa etária } \\
\hline 0 a 6 dias & 138 & 86,8 & 181 & 95,8 & 269 & 93,1 & 589 & 95,6 & 1295 & 95,0 \\
\hline 7 a 27 dias & 13 & 8,2 & 4 & 2,1 & 11 & 3,8 & 10 & 1,6 & 28 & 2,1 \\
\hline 28 a 364 dias & 8 & 5,0 & 4 & 2,1 & 9 & 3,1 & 17 & 2,8 & 37 & 2,7 \\
\hline 365 dias ou mais & 0 & 0,0 & 0 & 0,0 & 0 & 0,0 & 0 & 0,0 & 3 & 0,2 \\
\hline \multicolumn{11}{|l|}{ Sexo } \\
\hline Feminino & 84 & 52,8 & 89 & 47,1 & 133 & 46,0 & 293 & 47,6 & 629 & 46,1 \\
\hline Masculino & 74 & 46,5 & 94 & 49,7 & 150 & 51,9 & 294 & 47,7 & 674 & 49,4 \\
\hline Ignorado & 1 & 0,6 & 6 & 3,2 & 6 & 2,1 & 29 & 4,7 & 60 & 4,4 \\
\hline \multicolumn{11}{|l|}{ Raça/Cor } \\
\hline Branca & 39 & 24,5 & 44 & 23,3 & 68 & 23,5 & 94 & 15,3 & 241 & 17,7 \\
\hline Preta & 11 & 6,9 & 9 & 4,8 & 22 & 7,6 & 43 & 7,0 & 75 & 5,5 \\
\hline Amarela & 0 & 0,0 & 1 & 0,5 & 1 & 0,3 & 3 & 0,5 & 2 & 0,1 \\
\hline Parda & 57 & 35,8 & 76 & 40,2 & 100 & 34,6 & 243 & 39,4 & 604 & 44,3 \\
\hline Indígena & 0 & 0,0 & 0 & 0,0 & 1 & 0,3 & 0 & 0,0 & 0 & 0,0 \\
\hline Ignorado/Ausente & 52 & 32,7 & 59 & 31,2 & 97 & 33,6 & 233 & 37,8 & 441 & 32,4 \\
\hline \multicolumn{11}{|l|}{ Zona de residência } \\
\hline Urbana & 146 & 91,8 & 169 & 89,4 & 267 & 92,4 & 561 & 91,1 & 1244 & 91,3 \\
\hline Rural & 8 & 5,0 & 11 & 5,8 & 13 & 4,5 & 23 & 3,7 & 41 & 3,0 \\
\hline Periurbana & 2 & 1,3 & 1 & 0,5 & 3 & 1,0 & 2 & 0,3 & 2 & 0,1 \\
\hline Ignorado/ Ausente & 3 & 1,9 & 8 & 4,2 & 6 & 2,1 & 30 & 4,9 & 76 & 5,6 \\
\hline
\end{tabular}

Fonte: Cálculos realizados pela autora, 2016. Dados do SINAN: 2007 - 2015, obtidos na Secretaria de Estado de Saúde/MG.

Tabela 3. Distribuição dos casos de Sífilis Congênita segundo aspectos clínicos, no estado de Minas Gerais, 2007 a 2015. ( $\mathrm{n}=4.381)$.

\begin{tabular}{|c|c|c|c|c|c|c|c|c|c|c|}
\hline \multirow{2}{*}{ Ano } & \multicolumn{2}{|c|}{2007} & \multicolumn{2}{|c|}{2009} & \multicolumn{2}{|c|}{2011} & \multicolumn{2}{|c|}{2013} & \multicolumn{2}{|c|}{2015} \\
\hline & $\mathbf{N}$ & $\%$ & $\mathbf{N}$ & $\%$ & $\mathbf{N}$ & $\%$ & $\mathbf{N}$ & $\%$ & $\mathbf{N}$ & $\%$ \\
\hline \multicolumn{11}{|l|}{ Diagnóstico Clínico } \\
\hline Assintomático & 13 & 8,2 & 118 & 62,4 & 175 & 60,6 & 396 & 64,3 & 971 & 71,2 \\
\hline Sintomático & 2 & 1,3 & 23 & 12,2 & 47 & 16,3 & 91 & 14,8 & 222 & 16,3 \\
\hline Não se aplica & 1 & 0,6 & 9 & 4,8 & 14 & 4,8 & 44 & 7,1 & 67 & 4,9 \\
\hline Ignorado/Ausente & 143 & 89,9 & 39 & 20,6 & 53 & 18,3 & 85 & 13,8 & 103 & 7,6 \\
\hline \multicolumn{11}{|l|}{ Evolução do caso } \\
\hline Vivo & 148 & 93,1 & 170 & 89,9 & 249 & 86,2 & 507 & 82,3 & 1181 & 86,6 \\
\hline Óbito por SC & 3 & 1,9 & 6 & 3,2 & 6 & 2,1 & 13 & 2,1 & 38 & 2,8 \\
\hline Óbito por outras causas & 1 & 0,6 & 0 & 0,0 & 3 & 1,0 & 7 & 1,1 & 14 & 1,0 \\
\hline Aborto & 0 & 0,0 & 0 & 0,0 & 0 & 0,0 & 0 & 0,0 & 0 & 0,0 \\
\hline Natimorto & 0 & 0,0 & 3 & 1,6 & 14 & 4,8 & 31 & 5,0 & 43 & 3,2 \\
\hline Ignorado & 7 & 4,4 & 10 & 5,3 & 17 & 5,9 & 58 & 9,4 & 87 & 6,4 \\
\hline \multicolumn{11}{|l|}{ Diagnóstico final } \\
\hline SC recente & 137 & 86,2 & 184 & 97,4 & 275 & 95,2 & 585 & 95,0 & 1320 & 96,8 \\
\hline SC tardia & 0 & 0,0 & 2 & 1,1 & 0 & 0,0 & 0 & 0,0 & 0 & 0,0 \\
\hline Natimorto & 22 & 13,8 & 3 & 1,6 & 14 & 4,8 & 31 & 5,0 & 43 & 3,2 \\
\hline
\end{tabular}

Com a finalidade de facilitar a visualização da evolução temporal das características sociodemográficas e epidemiológicas, as tabelas 2 a 4 foram apresentadas em anos alternados.

Fonte: Cálculos realizados pela autora, 2016. Dados do SINAN: 2007 - 2015, obtidos na Secretaria de Estado de Saúde/MG. 
Verificou-se que o TNT no sangue periférico dos RN foi reagente em 3.115 casos (71,5\%). Já em relação ao TT, realizado após 18 meses do nascimento, houve $42,6 \%$ de incompletude da informação, impossibilitando a análise. No TNT realizado no líquor do RN, 139 casos $(3,2 \%)$ foram reagentes, 1.968 (44,9\%) não reagentes, e em $1.617(36,9 \%)$ casos não foram realizados (dados não apresentados em tabela).

Quanto à titulação ascendente, que é a comparação dos títulos da sorologia não treponêmica da criança após cada teste realizado durante o esquema de seguimento (VDRL aos 1, 3, 6, 12 e 18 meses de idade), 82 casos (1,9\%) tiveram elevação de título sorológico (titulação ascendente), 544 casos (12,4\%) não tiveram e 49,4\% não realizaram a comparação dos títulos. Em relação à evidência de T. pallidum em exame microscópico de material colhido em placenta, lesões cutaneomucosas, cordão umbilical ou necropsia, 111 $(2,5 \%)$ casos tiveram evidência, 701 (16\%) casos não tiveram, e não foi realizado exame em 1.866 $(42,6 \%)$ casos, houve incompletude dessa informação para $38,9 \%$ dos casos (dados não apresentados em tabela).

Quanto às alterações em relação ao exame do líquor, caracterizadas por celularidade maior que $10 /$ campos e proteinorraquia maior que 100 $\mathrm{mg} / \mathrm{ml}$, no recém-nato, $1.856(42,4 \%)$ casos não apresentaram alterações e 193 (4,4\%) apresentaram. Ainda, segundo dados laboratoriais, 2,7\% tiveram diagnóstico radiológico (exame dos ossos longos) com alterações ósseas observáveis aos raios-X $(\mathrm{Rx})$, tais como osteocondrite, osteoartrite, periostite (diafisite produtiva), rarefação óssea, tíbia em "lâmina de sabre" e 48,0\% casos estavam isentos de alterações ósseas observáveis (dados não apresentados em tabela).

A maioria não apresentou sinais e sintomas ao exame físico no momento da notificação, 2.978 não apresentaram icterícia $(68,0 \%)$; eram isentos de rinite $(75,3 \%)$; anemia $(73,9 \%)$, esplenomegalia $(73,4 \%)$, hepatomegalia $(73,1 \%)$, osteocondrite $(74,5 \%)$, lesões cutâneas $(74,0 \%)$ e pseudoparalisia $(74,9 \%)$.

A faixa etária predominante das mães foi de 20 a 39 anos (72,1\%). O PN foi realizado por $82,2 \%$ das gestantes. Receberam o diagnóstico de sífilis durante o PN, 2.463 (56,2\%) gestantes, enquanto que $1.233(28,1 \%)$ receberam no momento do parto ou curetagem (Tabela 4).

O TNT no momento do parto ou curetagem foi reagente para $3.880(88,6 \%)$ gestantes. Já, o TT confirmatório no parto ou curetagem, foi reagente para $1.299(29,7 \%)$ gestantes, e não realizado em $2.063(47,1 \%)$. Quanto ao esquema de tratamento foi inadequado para $2.418(55,2 \%)$ gestantes e adequado apenas para $203(4,6 \%)$ casos, sendo considerável a proporção de não realizados $(25,9 \%)$. A maioria dos parceiros sexuais não foi tratada concomitantemente $(55,8 \%)$, trataram oportunamente 587 (13,4\%) casos (Tabela 4).

Observou-se incremento na tendência temporal da taxa de incidência da SC no período entre 2007 e 2015, com variação anual da taxa de 30,6\% (IC95\%: 21,0 - 41,0) (Figura 1).

\section{Discussão}

A completude da informação propicia a caracterização da população estudada e o planejamento de ações para o enfrentamento na saúde pública, pois permite identificar os nós críticos. A qualidade e a pertinência das informações produzidas, com intuito de conhecer as condições de saúde da população, podem estar comprometidas quando se verificam variáveis com preenchimento inadequado ${ }^{17}$. Magalhães et al. ${ }^{18}$ salientam sobre a importância de registros referentes às notificações e ao acompanhamento de casos, porém a subnotificação, o preenchimento incompleto e/ ou informações conflituosas ou ignoradas foram observadas em parte significativa dos registros consultados em estudo realizado com gestantes VDRL reagentes em maternidades públicas de hospitais do Distrito Federal entre novembro de 2009 e dezembro de 2010.

A incompletude de variáveis dos SIS também foi observada nos estudos, que avaliaram a qualidade dos dados de notificação do SINAN de gestantes HIV positivas do Estado do Ceará entre 2000 e $2009^{19} \mathrm{e}$ dos agravos relacionados ao trabalho no município de Betim, MG, entre 2007 e $2011^{20}$. Uma possibilidade para o descompromisso com o preenchimento adequado pode ser a quantidade expressiva de campos das fichas de notificação/investigação do SINAN e o excesso de formulários para preenchimento nos serviços de saúde.

O SINAN agrega informações sobre diversas características dos agravos relacionados à SC, importantes para orientar a atuação da assistência e vigilância em saúde, serviços de vigilância epidemiológica e saúde materno-infantil, contribuindo para o planejamento de ações que busquem a erradicação ou a diminuição da infecção no Brasil. Apesar da prerrogativa de que todos os campos devam ser preenchidos, os mecanismos dos SIS que reforçam essa obrigatoriedade atin- 
Tabela 4. Distribuição do perfil clínico-epidemiológico das mães dos recém-nascidos com Sífilis Congênita, no estado de Minas Gerais, 2007 a 2015. $(\mathrm{N}=4.381)$.

\begin{tabular}{|c|c|c|c|c|c|c|c|c|c|c|}
\hline & \multicolumn{2}{|c|}{2007} & \multicolumn{2}{|c|}{2009} & \multicolumn{2}{|c|}{2011} & \multicolumn{2}{|c|}{2013} & \multicolumn{2}{|c|}{2015} \\
\hline & $\mathbf{N}$ & $\%$ & $\mathbf{N}$ & $\%$ & $\mathbf{N}$ & $\%$ & $\mathbf{N}$ & $\%$ & $\mathbf{N}$ & $\%$ \\
\hline \multicolumn{11}{|l|}{ Idade da mãe } \\
\hline 10 a 14 anos & 0 & 0,0 & 0 & 0,0 & 2 & 0,7 & 6 & 1,0 & 16 & 1,2 \\
\hline 15 a 19 anos & 29 & 18,2 & 41 & 21,7 & 67 & 23,2 & 160 & 26,0 & 336 & 24,7 \\
\hline 20 a 39 anos & 121 & 76,1 & 140 & 74,1 & 208 & 72,0 & 430 & 69,8 & 948 & 69,6 \\
\hline 40 a 59 anos & 3 & 1,9 & 8 & 4,2 & 9 & 3,1 & 13 & 2,1 & 24 & 1,8 \\
\hline Ausente & 6 & 3,8 & 0 & 0,0 & 3 & 1,0 & 7 & 1,1 & 39 & 2,9 \\
\hline \multicolumn{11}{|l|}{ Realizou pré-natal na gestação } \\
\hline $\operatorname{Sim}$ & 136 & 85,5 & 162 & 85,7 & 232 & 80,3 & 484 & 78,6 & 1145 & 84,0 \\
\hline Não & 18 & 11,3 & 23 & 12,2 & 43 & 14,9 & 99 & 16,1 & 139 & 10,2 \\
\hline Ignorado & 5 & 3,1 & 4 & 2,1 & 14 & 4,8 & 33 & 5,4 & 79 & 5,8 \\
\hline \multicolumn{11}{|l|}{ Diagnóstico de sífilis materna } \\
\hline Durante o pré-natal & 83 & 52,2 & 98 & 51,9 & 147 & 50,9 & 333 & 54,1 & 829 & 60,8 \\
\hline No momento do parto/curetagem & 48 & 30,2 & 54 & 28,6 & 87 & 30,1 & 188 & 30,5 & 345 & 25,3 \\
\hline Após o parto & 16 & 10,1 & 30 & 15,9 & 45 & 15,6 & 73 & 11,9 & 103 & 7,6 \\
\hline Não realizado & 3 & 1,9 & 2 & 1,1 & 1 & 0,3 & 5 & 0,8 & 7 & 0,5 \\
\hline Ignorado & 9 & 5,7 & 5 & 2,6 & 9 & 3,1 & 17 & 2,8 & 79 & 5,8 \\
\hline \multicolumn{11}{|c|}{ Teste não treponêmico no parto/curetagem } \\
\hline Reagente & 125 & 78,6 & 160 & 84,7 & 255 & 88,2 & 562 & 91,2 & 1201 & 88,1 \\
\hline Não reagente & 13 & 8,2 & 12 & 6,3 & 14 & 4,8 & 19 & 3,1 & 69 & 5,1 \\
\hline Não realizado & 14 & 8,8 & 10 & 5,3 & 7 & 2,4 & 16 & 2,6 & 50 & 3,7 \\
\hline Ignorado & 7 & 4,4 & 7 & 3,7 & 13 & 4,5 & 19 & 3,1 & 43 & 3,2 \\
\hline \multicolumn{11}{|c|}{$\begin{array}{l}\text { Teste confirmatório treponêmico no parto/ } \\
\text { curetagem }\end{array}$} \\
\hline Reagente & 41 & 25,8 & 31 & 16,4 & 71 & 24,6 & 162 & 26,3 & 530 & 38,9 \\
\hline Não reagente & 8 & 5,0 & 3 & 1,6 & 11 & 3,8 & 34 & 5,5 & 59 & 4,3 \\
\hline Não realizado & 74 & 46,5 & 117 & 61,9 & 148 & 51,2 & 301 & 48,9 & 543 & 39,8 \\
\hline Ignorado & 36 & 22,6 & 38 & 20,1 & 59 & 20,4 & 119 & 19,3 & 231 & 16,9 \\
\hline \multicolumn{11}{|l|}{ Esquema de tratamento } \\
\hline Adequado & 13 & 8,2 & 8 & 4,2 & 14 & 4,8 & 28 & 4,5 & 64 & 4,7 \\
\hline Inadequado & 62 & 39,0 & 92 & 48,7 & 127 & 43,9 & 327 & 53,1 & 829 & 60,8 \\
\hline Não realizado & 47 & 29,6 & 59 & 31,2 & 83 & 28,7 & 164 & 26,6 & 321 & 23,6 \\
\hline Ignorado & 37 & 23,3 & 30 & 15,9 & 65 & 22,5 & 97 & 15,7 & 149 & 10,9 \\
\hline \multicolumn{11}{|c|}{ Parceiro tratado concomitantemente à gestante } \\
\hline Sim & 21 & 13,2 & 20 & 10,6 & 33 & 11,4 & 78 & 12,7 & 211 & 15,5 \\
\hline Não & 70 & 44,0 & 101 & 53,4 & 155 & 53,6 & 360 & 58,4 & 818 & 60,0 \\
\hline Ignorado & 68 & 42,8 & 68 & 36,0 & 101 & 34,9 & 178 & 28,9 & 334 & 24,5 \\
\hline
\end{tabular}

Com a finalidade de facilitar a visualização da evolução temporal das características sociodemográficas e epidemiológicas, as tabelas 2 a 4 foram apresentadas em anos alternados.

Fonte: Cálculos realizados pela autora, 2016. Dados do SINAN: 2007 - 2015, obtidos na Secretaria de Estado de Saúde/MG.

gem apenas alguns campos como obrigatórios ${ }^{20}$. A maioria das variáveis, que compõe os formulários de notificação, é essencial, ou seja, são necessárias à investigação do caso, de sua cadeia epidemiológica e para o cálculo de indicadores epidemiológicos ou operacionais. O volume de dados para preenchimento, somados à ausência da obrigatoriedade no momento da digitação das informações, concorre para a falta de preenchimento ou pela opção de informação ignorada por parte dos profissionais de saúde ${ }^{20,21}$.

A incidência de SC, no estado de Minas Gerais, no ano de 2015 foi de 5,08 casos/1000 NV, superior à preconizada pelo MS, que determina 


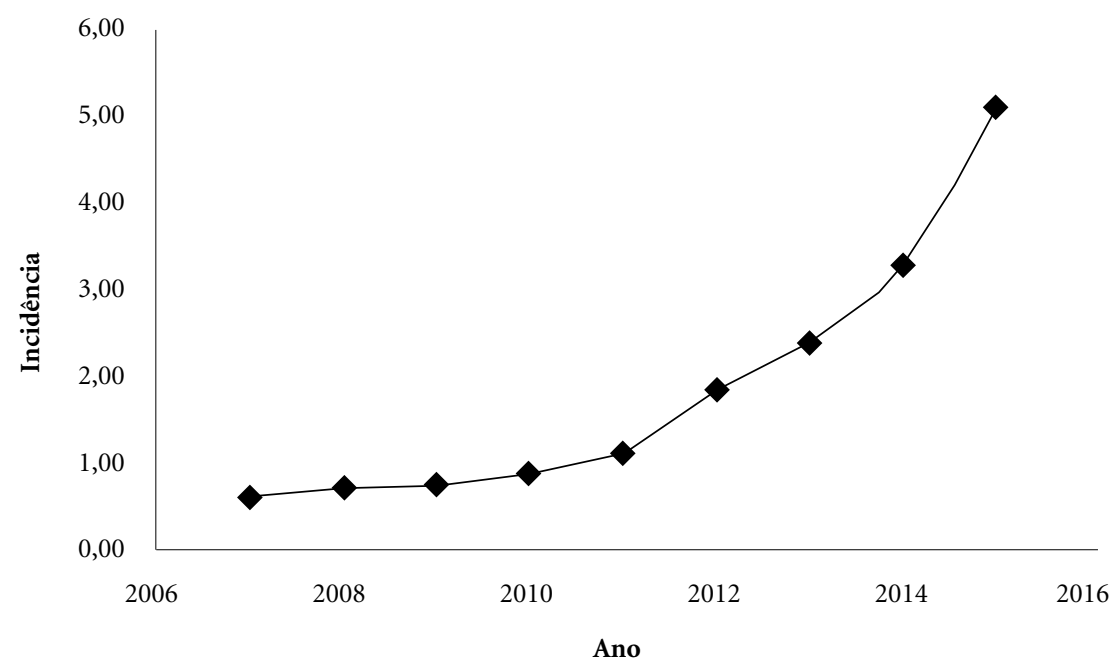

\begin{tabular}{cccc}
\hline Incidência de sífilis congênita & Porcentagem de variação anual & Intervalo de Confiança (95\%) & Tendência \\
\hline Incidência & 30,62 & $21,03-40,96$ & Ascendente
\end{tabular}

Figura 1. Série temporal (anual) da incidência dos casos notificados de SC ajustada pelo modelo de regressão linear generalizada de Prais-Winsten, no estado de MG, entre 2007 e 2015.

Fonte: Do autor, 2016.

a redução para $\leq 0,5$ caso/1.000 NV $\mathrm{N}^{12}$. Observouse aumento progressivo em todo período, o que corrobora com os estudos realizados no Estado de Goiás, entre 2009 e $2012^{22}$ e no Estado do Rio Grande do Sul entre 2001 e $2012^{23}$. Estudo realizado em Alagoas ${ }^{24}$ observou aumento da incidência de 41,3\% em 2011 (6,5 casos/1.000 NV), em relação à incidência média do período entre 2007 e 2011 (4,8 casos/1.000 NV). Isto evidencia um efetivo aumento no número de casos ou uma melhora das informações devido à redução de subnotificações ${ }^{24}$. Para Magalhães et al. ${ }^{18}$, uma assistência pré-natal deficiente leva a falhas no tratamento de gestantes com sífilis e pode resultar em um aumento do número de casos da SC. Ainda para os autores ${ }^{18}$, embora a SG não seja um agravo restrito às camadas menos favorecidas, a pouca escolaridade e a baixa renda podem ser marcadores importantes do pouco acesso aos serviços de saúde.

Houve aumento da incidência em outras regiões do Brasil, como na Região Sul entre 2001 e $2009^{25}$ e no município de Natal, no Rio Grande do Norte, entre 2004 e $2007^{26}$. Em estudo realizado nos Estados Unidos, no período de 2012 a
2014, houve também aumento da incidência ${ }^{27}$. Para Bowen et al. ${ }^{27}$, uma porcentagem substancial do incremento de casos é atribuível à deficiência na assistência pré-natal. E mesmo entre aquelas que receberam algum cuidado pré-natal, a detecção e o tratamento da sífilis materna ocorreu tardiamente para prevenir a SC. Estudo realizado na Colômbia observou que, a incidência aumentou de 2,15/1.000 NV em 2005 para 3,28/1.000 NV em 2011, os autores salientam também que esse aumento reflete falhas na execução sistemática das ações de promoção da saúde materno-infantil, indicando um problema real no acesso e qualidade do pré-natal ${ }^{28}$. Já em estudo realizado no México entre 1990 e 2009 observou-se incremento de $51,6 \%$ de $\operatorname{casos}^{29}$.

Ao contrário, estudos em outros países mostraram declínio no número de casos. Em estudo realizado na China, a incidência anual diminuiu de 1,15/1.000 NV em 2002 para 0,10/1.000 NV em $2011^{30}$. No Reino Unido no período de 2010 a 2015, houve diminuição da incidência de 0,0149 casos/1.000 NV em 2010 para 0,0013 casos/1.000 $\mathrm{NV}$ em $2014^{31}$. No Brasil, houve também declínio da incidência em algumas regiões. Em Campo 
Grande - Mato Grosso do Sul, a análise comparativa da incidência verificou que o número de SC em 2011 (5,85 casos/1.000 NV) foi inferior a 2006 (23,4 casos/1.000 NV $)^{32}$. Já no estado do Rio Grande do Norte a taxa de incidência foi de 4,3 e 0,9 nos anos de 2008 e 2010, respectivamente ${ }^{6}$.

No estado de MG houve aumento da tendência temporal da incidência com variação anual de $30,6 \%$. Esse cenário também pode ser observado em estudo realizado no estado de Mato Grosso entre 2001 e $2011^{33}$ e em Aracaju/SE entre 2008 e $2012^{34}$. No Brasil, nos últimos cinco anos, observou-se um aumento constante no número de casos de sífilis em gestantes, congênita e adquirida, que pode ser atribuído, em parte, pelo aprimoramento do sistema de vigilância epidemiológica, assim como o aumento da cobertura de testagem sorológica, com a ampliação do uso de testes rápidos, redução do uso de preservativo, resistência dos profissionais de saúde à administração da penicilina na Atenção Básica, desabastecimento mundial de penicilina, entre outros ${ }^{12}$.

A evolução temporal crescente para os casos de SC é sugestiva de melhoria na notificação ao longo dos anos. A subnotificação pode ser considerada um indicador indireto da fragilidade da assistência à saúde prestada ${ }^{33}$. A persistência de alta incidência da doença e taxas de transmissão vertical, mesmo após o aumento considerável da cobertura de assistência PN e do número médio de consultas com a implementação do SUS, indica que a qualidade do cuidado deve ser melhora$\mathrm{da}^{35}$. Serruya et al. ${ }^{36}$ salientam, em estudos realizados nos países da América Latina e Caribe, que para eliminar a SC é necessário um compromisso político de alto nível e com o apoio do MS.

Estudos realizados no Rio Grande do Norte $^{6} \mathrm{e}$ Sul do Brasil ${ }^{25}$ observaram maior prevalência até 6 dias de vida, 96,7\% e 94,9\%, respectivamente, corroborando com o presente estudo. O que infere tempo hábil no preenchimento da notificação logo após o nascimento. O diagnóstico de SC do recém-nascido feito em tempo oportuno, até os 6 dias de vida, permite a possibilidade de início do tratamento precoce ${ }^{37}$. Quanto à zona de residência, os resultados foram semelhantes a estudos realizados no estado do Rio Grande do Norte $(83,4 \%)^{6}$ e em Campo Grande - Mato Grosso do $\mathrm{Sul}(91,3 \%)$ onde a maioria das gestantes infectadas residiam em área urbana ${ }^{32}$.

Analisando a distribuição dos casos de SC, segundo dados laboratoriais, verificou-se que o TNT no sangue periférico do RN foi reagente para $71,5 \%$ casos, superior ao observado no município de Natal - Rio Grande do Norte, entre
2004 e 2007, com percentual de 51,1\% $\%^{26}$. Resultados inferiores ao do estado de Minas Gerais foram observados para alterações ao exame do líquor, resultados com ausência de alterações e exames não realizados.

Em relação às alterações ósseas observáveis no exame de $\mathrm{Rx}, 2,7 \%$ tiveram alterações e 48,0\% não. Estudo ${ }^{38}$ verificou consonância na ausência de alterações radiológicas em sua maioria $(57,4 \%)$. Nos casos suspeitos de SC, a radiografia dos ossos longos pode oferecer auxílio diagnósti$\mathrm{CO}^{26}$. Justifica-se a realização do exame nos casos suspeitos, uma vez que de 4 a 20\% dos recémnascidos infectados, a única alteração encontrada seja a radiográfica ${ }^{33}$.

Quanto ao diagnóstico definitivo, 95,2\% dos casos foram SC recente, semelhante à estudos realizados na região sul do Brasil entre 2001 e 2009 $(86,9 \%)^{25}$ e na região do Amazonas entre 2007 e $2009(83,3 \%)^{39}$. A SC recente surge até o segundo ano de vida e deve ser diagnosticada por meio de uma avaliação epidemiológica criteriosa da situação materna e de avaliações clínica, laboratorial e de estudos de imagem na criança ${ }^{33}$. A maioria das crianças com SC recente é assintomática ao nascer; portanto, o diagnóstico nem sempre é obvio e depende de alta suspeição clínica para a investigação da história materna, além de exames minuciosos na criança exposta ${ }^{11}$.

Analisando a distribuição do perfil epidemiológico e clínico das mães, verificou-se que $82,2 \%$ realizaram $\mathrm{PN}$ na gestação, corroborando com outros estudos $1,6,26,34$. Apesar da maioria das mães terem realizado $\mathrm{PN}$, um número expressivo obteve o desfecho para SC que é um indicador sentinela da qualidade da assistência ao PN. A notificação do agravo deve servir para desencadear uma investigação oportuna das causas do evento junto às unidades e profissionais de saúde, bem como junto às gestantes, de forma a corrigir falhas no programa de prevenção da doença ${ }^{2}$.

A maioria recebeu o diagnóstico de sífilis materna durante o PN (56,2\%), enquanto 39,1\% receberam no momento do parto/curetagem ou após o parto, divergente do estudo realizado em Aracaju - Sergipe entre 2008 e 2012, em que $73,5 \%$ das gestantes foram diagnosticadas apenas no momento do parto/curetagem ou após o parto e $26,5 \%$ durante a gestação ${ }^{34}$ e do estudo na cidade de Montes Claros, MG, entre 2007 e 2013, que evidenciou que a maioria das gestantes fez o PN no início do primeiro trimestre, porém com persistência do diagnóstico tardio ${ }^{40}$.

A dificuldade de prevenção da transmissão vertical permanece no entrave do diagnóstico e 
do tratamento precoce e adequado ${ }^{34}$. Infere-se que a gestante, ao receber o diagnóstico de sífilis durante a gestação, possibilita que a infecção ao concepto não evolua para SC se tratada adequadamente. Considera-se tratamento adequado da gestante quando este é realizado com penicilina e que tenha sido concluído 30 dias antes do parto, utilizando dose correta da medicação conforme estágio da doença e considerando o fato de o parceiro sexual ser devidamente medicado concomitantemente ${ }^{40,41}$.

A atenção ao $\mathrm{PN}$ visa acolher a mulher desde o início da gravidez de forma qualificada e humanizada, adotando como objetivo primordial ofertar condutas acolhedoras e com intervenções oportunas. No que se refere ao TNT no momento do parto ou curetagem, foi reagente para $88,6 \%$ dos casos, semelhante ao estudo realizado em Alagoas entre 2007 e $2011^{24}$. Os resultados encontrados remetem a um questionamento quanto à qualidade do PN ofertado, uma vez que o número de gestantes que realizaram $\mathrm{PN}$, apresentou ascensão tanto quanto a elevação do número de casos de SC notificados, indicando falhas na realização de exames oportunos.

$\mathrm{O}$ enfermeiro tem papel fundamental na prevenção da SC, pois ao atuar na captação precoce da gestante tem a possibilidade de realizar o teste rápido e ou solicitar o exame sorológico, além de realizar a busca ativa ao parceiro e acompanhamento do tratamento adequado do casal. O controle da SC requer maior comprometimento dos profissionais que atuam na atenção primária, já que é nesse nível de assistência que se espera que ocorra o acompanhamento $\mathrm{PN}$, solicitação precoce do exame de VDRL e os primeiros cuidados relacionados à prevenção da transmissão vertical ${ }^{42}$.

Quanto ao esquema de tratamento dessas mães, apenas 4,6\% realizaram adequadamente, $55,2 \%$ inadequadamente e $25,9 \%$ não realizaram. Em relação aos parceiros das gestantes, $55,8 \%$ não foram tratados concomitantemente a estas. Em estudo realizado em Aracaju - Sergipe entre 2008 e 2012, a maioria das gestantes também não realizou tratamento (50,8\%), 47,3\% realizaram inadequadamente e apenas 3,8\% o realizaram de forma adequada, com $84,6 \%$ dos parceiros não tratados concomitantemente ${ }^{34}$. Para que o tratamento da gestante com sífilis seja considerado adequado e assegure que não haja a possibilidade de transmissão vertical, a mulher deverá ser medicada com penicilina $\mathrm{G}$ benzatina (nas doses apropriadas à fase de infecção), o parceiro deverá ser tratado simultaneamente, e ela deve ser acompanhada mensalmente e finalizar o tratamento até 30 dias antes do parto ${ }^{43}$.

Reconhecendo a limitação de utilizar fontes de dados secundários, especialmente no que se refere a prováveis subregistros, subnotificações, erros de classificação e preenchimento, além de restringir as variáveis selecionadas ao que já foi coletado, identificou-se um número crescente de casos notificados de SC no período, e obteve-se informações pertinentes ao planejamento e organização dos serviços de saúde em relação à doença no estado. A incompletude das fichas foi uma limitação deste estudo, assim como a falta de acesso aos pacientes/prontuários para complementar informações, por ser um banco de dados de abrangência estadual.

\section{Conclusão}

A tendência temporal da incidência da SC foi crescente no período, que pode estar relacionado a maior sensibilidade do sistema de notificação, no que tange ao registro dos casos, ao tratamento inadequado pela maioria das gestantes e à pouca adesão do parceiro ao tratamento. Pois apesar do número de consultas do $\mathrm{PN}$ adequadas, houve expressiva quantidade com desfecho para SC, indicando falhas na assistência. Embora o agravo seja de fácil prevenção e de tratamento eficaz, ainda há um longo caminho a ser percorrido para se alcançar a meta nacional de controle da doença. Os achados deste estudo são importantes uma vez que ressalta a necessidade de adequações de ações voltadas para a redução dos casos de sífilis congênita no estado de Minas Gerais. Para que ocorra mudança nesse cenário, é necessário que, além das esferas governamentais, os profissionais de saúde e toda a sociedade civil estejam envolvidos ativamente em ações que visem o empoderamento e o protagonismo na educação sexual de adolescentes e adultos, especialmente quanto à utilização de preservativos e outras práticas para a realização de sexo seguro. Faz-se necessário reforçar ações de saúde para que essa problemática possa ser amenizada, especialmente com a otimização das estratégias da assistência pré-natal e saúde da mulher. 


\section{Colaboradores}

PIC Alves e SS Castro: Concepção, análise e interpretação dos dados; Redação do artigo ou revisão crítica relevante do conteúdo intelectual; Aprovação final da versão a ser publicada. LM Scatena: análise de tendência temporal dos dados. Revisão crítica relevante do conteúdo intelectual; Aprovação final da versão a ser publicada. VJ Haas: organização do banco de dados e análise estatística. Revisão crítica relevante do conteúdo intelectual; Aprovação final da versão a ser publicada.

\section{Agradecimentos}

Secretaria do Estado da Saúde de Minas Gerais - Subsecretaria de Vigilância e Proteção à Saúde - Coordenadoria Estadual IST/AIDS e Hepatites Virais, pela disponibilização do banco de dados informatizado.

\section{Referências}

1. Costa CC, Freitas LV, Sousa DM, Oliveira LL, Chagas AC, Lopes MV, Damasceno AK. Sífilis congênita no Ceará: análise epidemiológica de uma década. Rev Esc Enferm USP 2013; 47(1):152-159.

2. Lima MG, Santos RF, Barbosa GJ, Ribeiro GS. Incidência e fatores de risco para sífilis congênita em Belo Horizonte, Minas Gerais, 2001-2008. Cien Saude Colet 2013; 18(2):499-506.

3. Kirkcaldy RD, Su JR, Taylor MM, Koumans E, Mickey T, Winscott M, Kenney K, Weinstock HS. Epidemiology of syphilis among hispanic women and associations with congenital syphilis, Maricopa County, Arizona. Sex Transm Dis 2011; 38(7):598-602.

4. Campos ALA, Araúgo MAL, Melo SP, Andrade RFV, Gonçalves MLC. Sífilis em parturientes: aspectos relacionados ao parceiro sexual. Rev bras ginecol obstet. 2012; 34(9):397-402.

5. Corrales SC. Importancia epidemiológica del diagnóstico temprano en el manejo de sífilis gestacional y congénita, falla terapéutica del tratamento secundaria a demora en el diagnóstico. Rev salud bosque 2013; 3(2):43-48.

6. Carvalho IS, Brito RS. Sífilis congênita no Rio Grande do Norte: estudo descritivo do período 2007-2010. Epidemiol Serv Saúde [Internet]. 2014 Jun [acessado 2018 Fev 01];23(2):287-294. Disponível em: http:// www.scielo.br/scielo.php?script=sci_arttext\&pi$\mathrm{d}=$ S2237-96222014000200287\&lng=en.

7. Magalhães DMS, Kawaguchi IAL, Dias A, Calderon IMP. A sífilis na gestação e a sua influência na morbimortalidade materno-infantil. Comum Ciênc Saúde 2011; 1(22):43-54.

8. Blencowe H, Cousens S, Kamb M, Berman S, Lawn JE. Lives saved tool supplement detection and treatment of syphilis in pregnancy to reduce syphilis related stillbirths and neonatal mortality. BMC Public Health 2011; 11(3 Supl.):S9.

9. Domingues RMSM, Szwarcwald CL, Souza Junior PRB, Leal MC. Prevalência de sífilis na gestação e testagem pré-natal: Estudo Nascer no Brasil. Rev Saude Publica 2014 Out [citado 2017 Ago 30];48(5):766-74. Disponível em: http://www.scielo.br/pdf/rsp/v48n5/ pt_0034-8910-rsp-48-5-0766.pdf

10. Melo NGDO, Melo Filho DA, Ferreira LOC. Diferenciais intraurbanos de sífilis congênita no Recife, Pernambuco, Brasil (2004-2006). Epidemiol serv saúde 2011; 20(2):213-222.

11. Brasil. Ministério da Saúde (MS). Protocolo Clínico e Diretrizes Terapêuticas Para Prevenção da Transmissão Vertical de HIV, Sífilis e Hepatites Virais. Brasília: MS; 2018. [citado 2018 Jun 05]. Disponível em: http:// www.aids.gov.br/pt-br/pub/2015/protocolo-clinico -e-diretrizes-terapeuticas-para-prevencao-da-transmissao-vertical-de-hiv

12. Brasil. Ministério da Saúde (MS). Sífilis 2017. [Internet]. Brasília: MS; 2017. [citado 2018 Jan 05]. (Boletim Epidemiológico, v.48, n.36). Disponível em: http://portalarquivos.saude.gov.br/images/pdf/2017/ novembro/13/BE-2017-038-Boletim-Sifilis-11-2017 -publicacao-.pdf 
13. Lima VC, Mororó RM, Martins MA, Ribeiro SM, Linhares MSC. Perfil epidemiológico dos casos de sífilis congênita em um município de médio porte no nordeste brasileiro. J Health Biol Sci [periódico na Internet].2017 [citado 2018 Jan 18];5(1):56-61. Disponível em: http://dx.doi.org/10.12662/2317-3076jhbs. v5i1.1012.p56-61.2017

14. Cardoso A, Griep R. Perfil epidemiológico da sífilis congênita no município de cascavel/PR no ano de 2015. Rev Thêma et Sci [periódico na Internet].2017 jun./jul. [citado 2017 Dez 20];7(1):[cerca de 6 p.]. Disponível em: http://www.themaetscientia.fag.edu. br/index.php/RTES/article/view/501/520.

15. Romero DE, Cunha CB. Avaliação da qualidade das variáveis sócio-econômicas e demográficas dos óbitos de crianças menores de um ano registrados no Sistema de Informações sobre Mortalidade do Brasil (1996/2001). Cad Saude Publica 2006; 22(3):673-684.

16. Antunes JLF, Cardoso MRA. Uso da análise de séries temporais em estudos epidemiológicos. Epidemiol. Serv. Saúde 2015; 24(3):565-576.

17. Correia LOS, Padilha BM, Vasconcelos SMV. Métodos para avaliar a completitude dos dados dos sistemas de informação em saúde do Brasil: uma revisão sistemática. Cien Saude Colet 2014; 19(11):4467-4478.

18. Magalhães DM, Kawaguchi IA, Dias A, Calderon IM. Sífilis materna e congênita: ainda um desafio. Cad Saude Publica 2013; 29(6):1109-1120.

19. Lima DJM, Chagas ACMA, Mendes IC, Oriá MOB, Aquino PS, Pinheiro AKB Completude e consistência dos dados de gestantes HIV positivas notificadas. Rev Enferm UERJ 2014; 22(3):321-326.

20. Alvares JK, Pinheiro PMM, Santos AF, Oliveira GL. Avaliação da completude das notificações compulsórias relacionadas ao trabalho registradas por município polo industrial no Brasil, 2007-2011. Rev bras epidemiol 2015; 18(1):123-136.

21. Brasil. Ministério da Saúde (MS). SINAN: Sistema de Informação de Agravos de Notificação. [Internet]. Brasília: MS; [2020]. [acessado 2017 Mar 07]. Disponível em: http://portalsinan.saude.gov.br/

22. Rezende EMA, Barbosa NB. A sífilis congênita como indicador da assistência de pré-natal no estado de Goiás. Rev APS 2015; 18(2):220-232.

23. Teixeira LO, Vitola C, Mendoza-Sassi RA, Belarmino, V. Tendência temporal e distribuição espacial da sífilis congênita no estado do Rio Grande do Sul entre 2001 e 2012. Cien Saude Colet 2016; 23(8):2587-2597.

24. Alves WA, Cavalcanti GR, Nunes FA, Teodoro WR, Carvalho LM, Domingos RS. Sífilis Congênita: epidemiologia dos casos notificados em Alagoas, Brasil, 2007 a 2011. Rev port saúde soc 2016; 1(1):27-41.

25. Serafim AS, Moretti GP, Serafim GS, Niero CV, Rosa MI, Pires MM, Simões PWTA. Incidence of congenital syphilis in the South Region of Brazil. Rev Soc Bras Med Trop [periódico na Internet].2014 Abr [acessado 2017 Mar 05];47(2):170-178. Disponível em: http:// www.scielo.br/scielo.php?script $=$ sci_arttext\&pi$\mathrm{d}=$ S0037-86822014000200170\&lng=pt\&nrm $=$ iso
26. Holanda MTCG, Barreto MA, Machado RMM, Pereira RC. Perfil epidemiológico da sífilis congênita no município de Natal, Rio Grande do Norte - 2004 a 2007. Epidemiol serv saúde 2011; 20(1 Supl.):69-78.

27. Bowen V, Su J, Torrone E, Kidd S, Weinstock H. Increase in incidence of congenital syphilis - United States, 2012-2014. Weekly 2015; 64(44):1241-1245.

28. Alzate-Granados JP, Sánchez-Bello NF, Amaya-Arias AC, Peralta-Pizza F, Eslava-Schmalbach J. Disparidades en la incidencia de sífilis congénita en Colombia 2005 a 2011: un estudio ecológico. Rev salud pública 2012; 14(6):965-977.

29. Reyna-Figueroa J, Esparza-Aguilar M, Hernández-Hernández LC, Fernández-Canton S, Richardson -Lopez Collada VL. Congenital syphilis, a reemergent disease in Mexico: its epidemiology during the last 2 decades. Sex Transm Dis 2011; 38(9):798-801.

30. Hong FC, Yang YZ, Liu XL, Feng TJ, Liu JB, Zhang CL, Lan LN, Yao MZ, Zhou H. Reduction in mother-tochild transmission of syphilis for 10 years in Shenzhen, China. Sex Transm Dis 2014; 41(3):188-193.

31. Simms I, Tookey PA, Goh BT, Lyall H, Evans B, Townsend $\mathrm{CL}$, Fifer $\mathrm{H}$, Ison C. The incidence of congenital syphilis in the United Kingdom: February 2010 to January 2015. BJOG [periódico na Internet] $2016 \mathrm{Mar}$ [acessado 2018 Jul 9];67(2). Disponível em: http://onlinelibrary.wiley.com/doi/10.1111/1471-0528.13950/ pdf

32. Figueiró-Filho EA, Freire SSA, Souza BA, Aguena GS, Maedo CM. Sífilis e gestação: estudo comparativo de dois períodos (2006 e 2011) em população de puérperas. DST j bras doenças sex transm 2012; 24(1):32-37.

33. Oliveira LR, Costa MR, Barreto FR, Pereira SM, Dourado I, Teixeira MG. Evaluation of preventative and control measures for congenital syphilis in State of Mato Grosso. Rev Soc Bras Med Trop [serial on the Internet]. 2014 June [acessado 2017 Mar 0];47(3):334-340. Disponível em: http://www.scielo.br/scielo.php?script=sci_arttext \&pid=S0037=86822014000300334-\&lngen\&nrm = iso

34. Santos GS, Oliveira LX, Guimarães AMDN, Nardello DM, Matos B, Carvalho, BID. Aspectos epidemiológicos da sífilis congênita associados à escolaridade materna. Rev enferm UFPE on line 2016; 10(8):28452852.

35. Domingues RMSM, Saraceni V, Hartz ZMA, Lea MC. Sífilis congênita: evento sentinela em saúde. Rev Saude Publica 2013; 47(1):147-157.

36. Serruya SJ, Duran P, Martinez G, Romero M, Caffe S, Alonso M, Silveira MF. Maternal and congenital syphilis in selected Latin America and Caribbean countries: a multi-country analysis using data from the perinatal information system. Sex health 2015; 12(2):164-169.

37. Signor M, Spagnolo LML, Tomberg JO, Gobatto M, Stofel NS. Distribuição espacial e caracterização de casos de sífilis congênita. Rev enferm UFPE on line [periódico na Internet] 2018. [acessado 2018 jul 14];12(2):398-406. Disponível em: https://doi. org/10.5205/1981-8963 -v12i2a230522p398-406-2018 
38. Cavalcante PM, Pereira RBL, Castro JGD. Syphilis in pregnancy and congenital syphilis in Palmas, Tocantins State, Brazil, 2007-2014. Epidemiol. Serv. Saúde 2017; 26(2):255-264.

39. Soeiro CMO, Miranda AE, Saraceni V, Santos MC, Talhari S, Ferreira LC. Syphilis in pregnancy and congenital syphilis in Amazonas State, Brazil: an evaluation using database linkage. Cad Saude Publica 2014; 30(4):715-723.

40. Lafetá KRG, Martelli Júnior H, Silveira MF, Paranaíba LMR. Sífilis materna e congênita, subnotificação e difícil controle. Rev bras epidemiol 2016; 19(1):63-74.

41. Brasil. Ministério da Saúde (MS). Diretrizes para o controle da sífilis congênita. Brasília: MS; 2005. (Série Manuais, v. 62)

42. Silva DMA, Araújo MAL, Silva RM, Andrade RFV, Moura HJ, Esteves ABB. Knowledge of healthcare professionals regarding the vertical transmission of syphilis in Fortaleza -CE, Brazil. Texto contexto - enferm. 2014; 23(2):278-285.

43. Brasil. Ministério da Saúde (MS). Diretrizes para o controle da síflis congênita: manual de bolso. Brasília: MS; 2006. [acessado 2017 Mar 07]. Disponível em: http://www.aids.gov.br/sites/default/files/manual_sifilis_bolso.pdf

Artigo apresentado em 30/03/2018

Aprovado em 25/09/2018

Versão final apresentada em 27/09/2018 